\title{
Herpes and polyoma family viruses in thyroid cancer (Review)
}

\author{
DIMITRIS P. STAMATIOU ${ }^{1,2^{*}}$, STAVROS P. DERDAS ${ }^{2 *}$, ODYSSEAS L. ZORAS ${ }^{1}$ and DEMETRIOS A. SPANDIDOS ${ }^{2}$ \\ ${ }^{1}$ Department of Surgical Oncology, University Hospital, University of Crete, Heraklion 71003; \\ ${ }^{2}$ Laboratory of Clinical Virology, University of Crete, Medical School, Heraklion 71409, Greece
}

Received December 15, 2015; Accepted January 26, 2016

DOI: $10.3892 / \mathrm{ol} .2016 .4144$

\begin{abstract}
Thyroid cancer is considered the most common malignancy that affects the endocrine system. Generally, thyroid cancer derives from follicular epithelial cells, and thyroid cancer is divided into well-differentiated papillary (80\% of cases) and follicular (15\% of cases) carcinoma. Follicular thyroid cancer is further divided into the conventional and oncocytic (Hürthle cell) type, poorly differentiated carcinoma and anaplastic carcinoma. Both poorly differentiated and anaplastic carcinoma can arise either de novo, or secondarily from papillary and follicular thyroid cancer. The incidence of thyroid cancer has significantly increased for both males and females of all ages, particularly for females between 55-64 years of age, from 1999 through 2008. The increased rates refer to tumors of all stages, though they were mostly noted in localized disease. Recently, viruses have been implicated in the direct regulation of epithelial-mesenchymal transition (EMT) and the development of metastases. More specifically, Epstein-Barr virus (EBV) proteins may potentially lead to the development of metastasis through the regulation of the metastasis suppressor, $\mathrm{Nm} 23$, and the control of Twist expression. The significant enhancement of the metastatic potential, through the induction of angiogenesis and changes to the tumor microenvironment, subsequent to viral infection, has been documented, while EMT also contributes to cancer cell permissiveness to viruses. A number of viruses have been identified to be associated with carcinogenesis, and these include lymphotropic herpesviruses, namely EBV and Kaposi's sarcoma-associated herpesvirus [KSHV, also known as human herpesvirus type 8 (HHV8)]; two hepatitis viruses, hepatitis B virus (HBV) and hepatitis $\mathrm{C}$ virus ( $\mathrm{HCV})$; human papillomaviruses (HPVs); human T cell lymphoma virus (HTLV); and a new polyomavirus, Merkel cell polyomavirus identified in
\end{abstract}

Correspondence to: Dr Stavros P. Derdas, Laboratory of Clinical Virology, University of Crete, Medical School, Heraklion 71409, Greece

E-mail: derdas@med.uoc.gr

*Contribured equally

Key words: herpesviruses, polyomaviruses, thyroid cancer
2008. In this review, we examined the association between thyroid cancer and two oncogenic virus families, the herpes and polyoma family viruses, and we discuss their potential role as causative agents in thyroid carcinogenesis.

\section{Contents \\ 1. Introduction \\ 2. Polyomaviruses \\ 3. Herpesviruses \\ 4. Conclusions and future perspectives}

\section{Introduction}

Thyroid cancer is the most common malignancy of the endocrine system $(1,2)$. The main types of thyroid cancer derive from follicular epithelial cells, including well-differentiated papillary ( $80 \%$ of cases) and follicular (15\% of cases) carcinoma, the latter being further divided into conventional and oncocytic (Hürthle cell) type, poorly differentiated carcinoma and anaplastic carcinoma (2-4). Both poorly differentiated and anaplastic carcinoma can arise either de novo, or secondarily from papillary thyroid cancer (PTC) and follicular thyroid cancer (FTC) (2-4). Patients with early stage well-differentiated papillary or follicular carcinoma usually have an excellent prognosis, unlike those with either aggressive tumors or distant metastases, who have a 5-year survival rate of $40 \%$ (5). Unlike the 3 aforementioned types of thyroid cancer, medullary cancer, derives from the neural crest, more specifically, the parafollicular, or C cells (6). It accounts for $3-4 \%$ of all thyroid cancer cases $(7,8)$, and its clinical course varies from indolent, to rather aggressive, with associated high mortality rates (6). Primary thyroid lymphoma represents a rare non-Hodgkin lymphoma (9), deriving predominantly from B-lymphocytes (10).

Thyroid cancer predisposition factors include a history of radiation exposure through either medical treatment, or fallout from nuclear accidents during childhood (11), thyroid nodules or goiter, as well as family history of thyroid cancer (11). From 1999 through 2008, the incidence of thyroid cancer has increased significantly for both individuals of both genders, and in particular for females aged between 55 and 64 years (12). These increased rates refer to tumors of all stages, although they have been moslty noted in localized disease (12). The reasons 
for this increase have not yet been completely elucidated (12). However, medical scrutiny, through ultrasound imaging and the fine needle aspiration cytological confirmation of small lesions that may have otherwise gone undiagnosed, has been proposed to account for the rising incidence rates $(13,14)$. The increased incidence of both small and large tumors across genders and multiple racial/ethnic groups suggests that the former factor does not solely drive this trend (12,15-17). Chronic lymphocytic thyroiditis constitutes the sole risk factor predisposing to primary thyroid lymphoma acquisition, being present in $50 \%$ of cases, and increasing the risk by 60 -fold $(18,19)$.

Recently, knowledge of the genetic alterations involved in thyroid cancer, has markedly increased $(1,2)$. More than $70 \%$ of PTCs contain BRAF, a serine-threonine kinase of the RAF protein family, and RAS, an intracellular G protein that propagates signals from receptor tyrosine kinases and $\mathrm{G}$ coupled receptors, point mutations, as well as RET/PTC, fusion between the 3 ' portion of the cell membrane receptor tyrosine kinase encoded by the RET proto-oncogene and the 5 ' portion of other unrelated genes, and $T R K$ rearrangements $(1,2,20-23)$. The above mutually exclusive mutations may potentially activate the mitogen-activated protein kinase (MAPK) pathway (1). As regards FTCs, mutually exclusive $R A S$ point mutations and $P A X 8 /$ peroxisome proliferator-activated receptor $\gamma(P P A R \gamma)$ rearrangements-from $\mathrm{t}(2 ; 3)(\mathrm{q} 13 ; \mathrm{p} 25)$ translocation resulting in fusion of the $P A X 8$ gene, coding for the thyroid-specific paired domain transcription factor (2), and the PPAR $\gamma$ gene (24) have been identified in $70-75 \%$ of cases (25). Poorly differentiated and anaplastic carcinomas contain mutations concerning phosphatidylinositol-3'-kinase (PI3K)/AKT, a serine threonine kinase that acts as a key effector of PI3K (26) signaling pathway, as well as mutations of TP53 and CTNNB1, encoding for $\beta$-catenin, the mutation of which is identified in $25 \%$ and $65 \%$ of poorly differentiated and anaplastic carcinomas, respectively (26), genes rarely found in well-differentiated papillary and follicular cancers (27). Even though the majority of medullary cancers are sporadic, almost $20 \%$ are hereditary, arising from a germline mutation in the RET proto-oncogene (6). Hereditary cases may either present isolated, or as part of multiple endocrine neoplasia syndrome type 2 (MEN2) (6).

The metastasizing ability of thyroid cancer is attributed to the induction of an epithelial-mesenchymal transition (EMT), that involves the invading ability of epithelial cells into the surrounding tissues (28). Despite the fact that oncogenic mutations have been associated with the induction of EMT, secondary factors are required (29). A number of viruses have been implicated in the direct regulation of EMT and in the development of metastases (29). More specifically, Epstein-Barr virus (EBV) proteins may potentially lead to the development of metastasis through the regulation of the metastasis suppressor, Nm23 (30), and the control of Twist expression (31). The significant enhancement of the metastatic potential, through the induction of angiogenesis and changes to the tumor microenvironment, subsequent to viral infection, has been documented (32), while EMT also contributes to cancer cell permissiveness to viruses (33).

Almost $20 \%$ of cancer cases have been linked to infectious factors, including viruses $(34,35)$. To date, 7 human viruses, strongly associated with carcinogenesis, have been identified and characterized as tumorigenic (34). These are
2 lymphotropic herpesviruses, namely EBV and Kaposi's sarcoma-associated herpesvirus [KSHV, also known as human herpesvirus type 8 (HHV8)]; 2 hepatitis viruses, hepatitis B virus and hepatitis $\mathrm{C}$ virus; human papillomaviruses (HPVs); human T cell lymphoma virus (HTLV); and a new polyomavirus (PyV), Merkel cell PyV (MCPyV) identified in 2008 (36).

This review examines the association between thyroid cancer and 2 oncogenic virus families, the herpes and polyoma family viruses, and discusses their potential role as causative agents in thyroid carcinogenesis.

\section{Polyomaviruses}

Overview. Since 1971, $10 \mathrm{PyVs}$ that infect humans have been identified, including JC virus (JCV) and BK virus (BKV) found over $>40$ years ago, as well as the recently isolated MCPyV, KI PyV (KIPyV), WU PyV (WUPyV), human PyV (HPyV)6, HPyV7, trichodysplasia spinulosa PyV (TSPyV), HPyV9 and MWPyV (37).

PyVs are obiquitous worldwide, with seroprevalence rates ranging from 35 to $90 \%(34,37)$. Apart from a productive life cycle with resultant cell lysis, often associated with minimal symptoms, PyVs can also establish a persistent infection; significant disease though, is limited to patients with immune system disfunction $(34,37,38)$. More specifically, JCV is associated with progressive multifocal leukoencephalopathy in HIV-AIDS, autoimmune diseases treated with certain lymphocyte-specific antibodies and hematological diseases; BKV with hemorrhagic cystitis following allogeneic hematopoietic stem cell transplantation and post-kidney transplantation PyV-associated nephropathy (37). TSPyV is linked to trichodysplasia spinulosa, a skin disease found in immune-impaired transplant patients that is characterized by virus-induced lytic and proliferative tumor-like features (37). KIPyV and WUPyV have been isolated from the respiratory tract, HPyV6 and HPyV7 from the skin, HPyV9 from the serum and skin, and MWPyV from stools and skin; However, none of these viruses have been associated with specific human pathology (37). MCPyV is the only PyV with a robust correlation with human cancer, more specifically Merkel cell carcinoma, a rare skin tumor found in elderly, chronically immunosupressed patients (37).

All PyVs have a similar morphological and functional pattern (39), with their virions consisting of non-enveloped icosahedral particles $40-45 \mathrm{~nm}$ in diameter, and a genome of $\sim 5 \mathrm{~kb}$ organized as circular double-stranded DNA wrapped around host cell-derived histones (37). This genome is divided into 3 functional parts: i) the non-coding control region (NCCR), which contains the origin of replication, the transcription start sites and promoter/enhancer elements, and regulates the expression of the early and late viral genes; ii) the early gene region, encoding the large T-antigen (LTag) and the small Tag which derives from a major transcript by alternative splicing and facilitate viral genome replication and transformation; and iii) the late gene region, encoding capsid proteins VP-1, VP-2 and VP-3, produced by alternative splicing of a primary transcript, and assembled in the nucleus to form the viral capsid (37).

Two possible outcomes may follow cell infection by PyV: i) in the case of a permissive host, viral entry results in viral DNA replication, followed by progeny virion production, and 
Table I. Investigation of polyomaviruses in thyroid tumors.

\begin{tabular}{|c|c|c|c|}
\hline Virus investigated & Investigated thyroid tissue & Method & Authors/(Refs.) \\
\hline SV40 & $\begin{array}{c}\text { PTC, MTC, NTT, AITD } \\
\text { (Hashimoto's thyroiditis), DTG }\end{array}$ & $\begin{array}{l}\text { PCR, Southern blotting, } \\
\text { immunohistochemistry }\end{array}$ & Pacini et al (44) \\
\hline SV40 & $\begin{array}{l}\text { PTC, ATC, MTC, NTT, AITD } \\
\text { (Grave's thyroiditis) }\end{array}$ & $\begin{array}{l}\text { PCR, RT-PCR, } \\
\text { immunohistochemistry }\end{array}$ & Vivaldi et al (45) \\
\hline SV40 & $\begin{array}{l}\text { PTC, Benign thyroid nodules } \\
\text { (including Hashimoto's thyroiditis), NTT }\end{array}$ & PCR & Ozdarendeli et al (46) \\
\hline BKV & PTC, MH, NTT & PCR & Stamatiou et al (47) \\
\hline
\end{tabular}

cell lysis; ii) in the case of a non-permissive host, an abortive infection or cell transformation, known as oncogenesis, ensues, characterized by the continued expression of the viral early genes (40-43). The starting point of this latter process, is the genetic and functional uncoupling of the early gene expression from the later steps of the viral life cycle, namely viral DNA replication, late gene expression, virion assembly and host cell lysis. Cell cycle control is subverted by the Tags through the inactivation of signal transduction pathways and the tumor suppressor proteins, pRB and p53, eventually leading to neoplastic transformation (37).

PyVs in thyroid cancer. To the best of our knowledge, few studies to date have investigated the association of PyVs with thyroid cancer (44-47), as shown in Table I. Three of these studies (44-46) detected sequences of simian vacuolating virus 40 (SV40), a virus that infected rhesus monkey kidney cells used for polio vaccine production (48), in thyroid gland specimens. Since SV40 has proven to be oncogenic in rodents $(37,38,44)$ and has transforming capacity in human cells in vitro (44), the wide distribution of SV40 contaminated vaccines in the early 1960 s, led to a concern for potential tumor induction in humans (37). A single study investigated the presence of BKV in post-operative thyroid specimens (47).

Most of our knowledge regarding the association of SV40 with thyroid cancer, is based on 3 studies examining the presence of SV40 DNA in thyroid specimens (44-46). The first study investigated the presence of SV40 sequences in 69 patients with PTCs using Southern blotting and polymerase chain reaction (PCR) (44). Seven normal peritumoral thyroid specimens, 4 blood specimens from patients with PTCs, 1 Hashimoto's thyroiditis, 5 toxic diffuse goiters, 3 medullary carcinomas and 9 breast carcinomas, were also studied. Southern blot analysis yielded positive results for 3 of the cases of PTC (4.3\%). PCR amplification followed by sequence analysis, confirmed the presence of SV40 sequences, including the 203 bp fragment of the aminoterminus of LTag, the 294 bp fragment of the VPl gene, as well as the 483 bp entire regulatory region, integrated in the tumoral DNA of the aforementioned samples. All the other samples scored negative for SV40 (44). Immunohistochemistry (IHC), performed for the LTag, revealed positive cytoplasmic staining in the 3 SV40-positive cases, not found in the negative controls (44). Another study (45), enroling 109 patients ( 80 females/29 males), performed by the same group, investigated the presence of SV40 DNA in a larger variety of thyroid pathologies, including 29 specimens of papillary cancer, 20 myeloid cancers, 20 anaplastic cancers and 20 specimens of Graves' disease. Specimens of normal thyroid tissue, 10 adjacent to papillary cancer, 10 adjacent to anaplastic cancer, and 20 from patients affected by multinodular goiter, were also included. Additionally, 20 peripheral blood mononuclear cell samples from relatives of patients with sporadic myeloid cancer, were analyzed. PCR amplification of the 172 bp N-terminal SV40 Tag and filter hibridization confirmed the presence of viral sequences in a percentage ranging from $66 \%$ in papillary cancers $(\mathrm{P}=0.02)$, to $100 \%$ in anaplastic cancers $(\mathrm{P}=0.01)$, while $90 \%$ of medullary cancer samples were positive $(\mathrm{P}=0.01)$. SV40 sequences were detected in a similar percentage, ranging from 60 to $100 \%$, in corresponding normal thyroid tissues next to the tumors, while the corresponding detection percentages in specimens taken from patients with multinodular goiter and Graves' disease, were 10 and 20\%, respectively. A total of $25 \%$ of blood samples were positive for SV40 Tag DNA (45). Positive samples were further investigated for the presence of the 314 and 294 bp sequences of the SV40 regulatory and VPl regions, respectively (45). SV40 sequence specificity, was confirmed by DNA sequencing. Subsequent RT-PCR, that was performed in the 24 thyroid cancer specimens yielded positive results for SV40 DNA, revealing mRNAs specific for SV40 Tag in 69\% (9/13) of PTCs, and 73\% (8/11) of anaplastic carcinomas. None of the 30 samples were found to be SV40-negative, used as negative controls, was positive. IHC was finally performed in the samples found positive by RT-PCR, while the 30 SV40-negative thyroid tissues were used as controls. A total of 33\% (3/9) of the PTCs and 100\% (8/8) of the anaplastic carcinomas were immunoreactive, and showed mainly cytoplasmic staining. The third study included 99 patients (21 males/78 females) with thyroid nodules, 8 of whom (8.08\%), were diagnosed with PTCs, while 91 (91.02\%) had benign thyroid nodules, 8 of which, harboured Hashimoto's thyroiditis (46). Both nodular and normal thyroid tissue was obtained from each patient. All 198 specimens were investi- 
gated for the presence of SV40, through the amplification of Tag coding sequences, using PCR (46). Sequences of SV40 were detected in 4 out of 99 nodules, 2 of which were PTCs, while the remaining were benign thyroid nodules. The presence of SV40 DNA in the thyroid nodules was confirmed by sequence analysis using the SV40P1 primer, and by cloning of the 243 bp PCR product into the Topo XL cloning vector (46).

A 95 bp sequence of the BKV VPl gene was investigated in frozen thyroid samples obtained from 30 patients with thyroid nodules (6 males/24 females) using PCR (47). A total of 14 out of the 30 patients $(46.7 \%)$ suffered from PTC, while 16/30 (53.3\%) had multinodular hyperplasia. Nodular, as well as adjacent normal tissues, were obtained from each patient. Taken as a whole, 18/30 (60.0\%) nodular tissue samples harboured BKV DNA, while the corresponding percentage in adjacent normal tissue was $43,3 \%$ (13/30). More specificaly, the VPl sequence was detected in 8/14 malignant specimens (57.1\%), compared to $6 / 14(42.8 \%)$ of adjacent normal tissue. The corresponding percentages for multinodular hyperplasia were $62.5 \%(10 / 16)$ and $43.7 \%$ (7/16), respectively (47).

\section{Herpesviruses}

Overview. Eight identified viruses from the herpesviridae family infect humans, categorized in 3 subfamilies, namely $\alpha$, $\beta$ and $\gamma$. The $\alpha$ subfamily includes herpes simplex viruses (HSV) type 1 and 2, as well as varicella zoster virus (VZV or HHV-3). Cytomegalovirus (CMV or HHV-5), and roseola viruses (HHV) 6 and 7 constitute the $\beta$ subfamily, while EBV (HHV-4) and KSHV/HHV8, belong to the $\gamma$ subfamily (49,50). Human herpesviruses are large (100-200 nm), enveloped, that contain double stranded DNA, enclosed in an icosahedral protein capsid (49).

As with PyVs, herpesviruses are widespread infectious agents, and almost $100 \%$ of adults have been infected in their lifetime (49). Lifelong infection is established, as the viral genome latently persists in the host cell nucleus (50). Although all herpesviruses infect epithelial cells (49), thus gaining access to the host, long-term residency and latency is strictly dictated by the specific herpes subfamily $(49,50)$, determined by the presence of cell-surface receptors, as well as intracellular conditions (49), $\alpha$ herpesviruses establish latency in neurons, $\beta$ herpesviruses in macrophages and lymphocytes, and $\gamma$ herpesviruses in lymphocytes alone $(49,50)$.

HSV-1 is associated with blisters on the lips, known as herpes labialis, while HSV-2 causes genital blisters (49). Both viruses may be associated with either lesion though (49). HSV may additionaly reach the eyes causing keratitis, potentially leading to blindness if left untreated (51), or may attack the brain, with resultant encephalitis or meningitis (52). Primary infection with VZV causes chickenpox, associated with a vesicular itchy rash primarily affecting the trunk and the head, while later reactivation is known as herpes zoster or shingles, limited to a specific body area (49). HHVs-6 and -7, known as roseola viruses, form skin rashes referred to as exanthema subitum (53). Congenital CMV infection may lead to birth defects (54), while mononucleosis, characterized by fever, sore throat, fatigue, as well as swolen lymph nodes, is the main disease associated with CMV in adolescents, though it is more commonly caused by EBV (49). Both $\gamma$ subfamily herpesviruses are tumorigenic agents (49). HHV-8 is mainly the etiologic agent associated with Kaposi's sarcoma, a cancer most frequently diagnosed in patients with AIDS (55). EBV is mainly associated with nasopharyngeal carcinoma, as well as with Burkitt, Hodgkin's, immune-suppression-related non-Hodgkin, and extranodal NK/T-cell lymphomas (56).

EBV represents the typical example of herpesviral strategy (49). Upon infection of a cell, the two ends of the initially linear viral genome, bind to each other, persisting in an episomal state, facilitating latency establishment $(57,58)$, as opposed to lytic activation, which requires genome linearization (59). No virions are produced during the latent state, allowing for the expression of only a few viral genes, which affect B-lymphocyte growth mechanisms, causing their immortalization (60). Six nuclear antigens, namely EpsteinBarr nuclear antigen (EBNA)1, -2, -3A, -3B, -3C, as well as the protein EBNA-LP; 3 membrane proteins, EBV latent membrane protein (LMP)1, -2A, -2B; 2 small nuclear RNAs, EBV-encoded small RNA (EBER)1 and -2, as well as BART region trancripts, encoding most of EBV's microRNAs, are associated with this latent infection of immortalized B cells (61). Conversely, the key role for the transition from the latent to the lytic cycle, inducing viral replication, is played by the BZLF1 and BRLF1 proteins (62).

Herpesviruses in thyroid cancer. Similar to PyVs, the association of members of the herpes family with thyroid cancer is based on detection methods of the presence of viral genes and gene products in thyroid tumor tissue $(29,47,63)$. The studies that investigate this association are presented in Table II. The majority of these studies refer to EBV (47,63-69), while 4 other members of the family have also been studied, although less extensively $(29,69)$.

The detection of EBV in thyroid cancer has proven controversial (47,63-69). The EBV persisting capacity in B-lymphocytes and its contribution to lymphoma formation (56), prompted the initial investigation of EBV in thyroid lymphomas (63-65), while subsequent studies investigated the presence and contribution of EBV in other types of thyroid malignancies (66-69).

Three studies examined the presence of EBV in thyroid lymphomas (63-65). EBV-related mRNA, as well as the associated proteins were investigated in 32 cases of thyroid lymphoma and 30 cases of Hashimoto's thyroiditis by in situ hybridization (ISH) and IHC on routinely processed tissue sections (63). Three cases of thyroid lymphoma demonstrated the presence of EBER. Gene products, BHLF1, LMP, and BZLF1 proteins were detected in 2 of the EBER-positive cases (63). The second study enrolled 30 patients with thyroid lymphoma and 28 with chronic lymphocytic thyroiditis (10 males/48 females) (64). Only 24 and 16 cases of thyroid lymphoma and chronic thyroiditis, respectively, were finally used, as the rest of the samples revealed poorly preserved DNA, based on the presence of the $\beta$-globin PCR band, and were thus excluded from further analysis (64). PCR amplification revealed positive EBV products in 1 case of chronic lymphocytic thyroiditis and 2 cases of thyroid lymphoma, while ISH yielded positive signals in the nucleus of tumor cells of only 1 of the lymhomas found positive by PCR, while LMP-1 was also expressed in the cytoplasm of lymphoma cells (64). The third and most 
Table II. Investigation of herpesviruses in thyroid tumors.

\begin{tabular}{|c|c|c|c|}
\hline Virus investigated & Investigated thyroid tissue & Method & Authors/(Refs.) \\
\hline EBV & TL, AITD (Hashimoto's thyroiditis) & $\begin{array}{l}\text { In situ hybridization, } \\
\text { immunohistochemistry }\end{array}$ & Takahashi et al (63) \\
\hline EBV & TL, CLTH & PCR, in situ hybridization & Tomita et al (64) \\
\hline EBV & $\mathrm{TL}$ & $\begin{array}{l}\text { In situ hybridization, } \\
\text { immunohistochemistry }\end{array}$ & Lam et al (65) \\
\hline EBV & $\begin{array}{l}\text { PTC, undifferenciated carcinoma, SCC, } \\
\text { AITD (Grave's disease), MH, NTT }\end{array}$ & $\begin{array}{c}\text { PCR, RT-PCR, in situ hybridization, } \\
\text { immunofluorescence }\end{array}$ & Shimakage et al (66) \\
\hline EBV & PTC, MH, NTT & PCR & Stamatiou et al (47) \\
\hline EBV & PTC & In situ hybridization & Kijima et al (67) \\
\hline EBV & WT & $\begin{array}{l}\text { PCR, in situ hybridization, } \\
\text { immunohistochemistry }\end{array}$ & Ludvikova et al (68) \\
\hline $\begin{array}{l}\text { EBV, CMV, HSV1, } \\
\text { HSV2, HHV8 }\end{array}$ & Benign thyroid tumors (not specified) & PCR, Southern hybridization & Tsai et al (69) \\
\hline HSV1, HSV2 & $\begin{array}{l}\text { AITD, FA, FTC, } \\
\text { FVPC, PTC, ATC }\end{array}$ & $\begin{array}{l}\text { PCR, immunohistochemical } \\
\text { staining, western blot analysis }\end{array}$ & Jensen et al (29) \\
\hline
\end{tabular}

EBV, Epstein-Barr virus; CMV, cytomegalovirus; HSV, herpes simplex virus; HHV8, human herpesvirus type 8 (also known as Kaposi's sarcomaassociated herpesvirus (KSHV); AITD, autoimmune disease; FA, follicular adenoma; FTC, follicular thyroid carcinoma; PTC, papillary thyroid carcinoma; FVPC, follicular variant of papillary thyroid carcinoma; ATC, anaplastic cancer; TL, thyroid lymphoma; CLTH, chronic lymphocytic thyroiditis; SCC, squamous cell cancer; MH, multinodular hyperplasia; NTT, normal thyroid tissue; WT, warthin-like tumor; PCR, polymerase chain reaction.

recent study, analyzed the clinicopathological characteristics of primary and secondary thyroid lymphomas affecting the Hong Kong Chinese population over a period of 3 decades, and investigated the expression of EBV genes, using ISH and IHC (65). A total of 23 patients with primary disease, 15 of whom had Hashimoto's thyroiditis, as well as 9 patients with secondary disease were enroled. EBV mRNAs were detected in 1 primary and 1 secondary thyroid lymphoma (65).

EBV has been suggested to contribute to thyroid tumor progression, defined as the pathological dedifferentiation of tumor cells, leading to a more undifferentiated tumor type (66). To examine the potential involvement of EBV expression in the progression of thyroid cancer, 10 PTCs, normal tissue specimens at the peripheral region of 4 of the PTCs, 11 undiferentiated carcinomas, 1 thyroid squamous cell carcinoma (SCC), as well as negative controls including 2 thyroid nodular hyperplasia and 2 Graves' disease specimens, all taken from Japanese patients were used. Specimens were subjected to PCR, RT-PCR, mRNA ISH and indirect immunofluorescence staining. PCR was performed in 13 of the samples, including 9 PTCs, 3 undifferentiated carcinomas and 1 SCC, all of which showed amplified EBV DNA in the region of BamHIW. ISH for the detection of EBV mRNAs expression was performed using BamHIW, EBER1 and EBNA2 probes, while immunofluorescence for the detection of EBV proteins was performed using 4 monoclonal antibodies, namely anti-EBNA2, anti-LMP1, anti-BZLF1 and anti-CD21. Despite the fact that EBV infection, and mRNA and protein expression was detected in all carcinoma specimens, irrespective of the degree of histological differentiation, both mRNA and protein exression was much more prominent in the undifferentiated carcinomas. Both the normal thyroid tissue specimens, as well as the nodular hyperplasia and Graves' disease specimens used as controls, showed no, or very few signals during ISH with any of the probes (66).

A similarly high EBV detection percentage in thyroid nodules consisting of PTCs, and multinodular hyperplasia specimens, as well as adjacent normal thyroid tissues, was recently reported in a previously mentioned study (47). The investigation included the detection of 161, 168 and $118 \mathrm{bp}$ sequences of the $L M P 1, E B N A 2$ and EBER 1 genes, respectively. As regards $L M P 1$, the sequence was detected in $50 \%(15 / 30)$ of the nodules, and in $46.7 \%(14 / 30)$ of the adjacent normal tissues. More specifically, 57.1\% (8/14) of the PTCs and $43.8 \%$ (7/16) of multinodular hyperplasia specimens harboured the sequence, while the percentages of the adjacent normal tissues were $35.7 \%(5 / 14)$ and $56.2 \%(9 / 16)$, respectively. Much higher percentages considering EBNA2 sequence detection, were found in the study, although similar between nodular (90\%-27/30) and adjacent normal (90-27/30) tissue. The vast majority of both malignant $(92.9 \%, 13 / 14)$ as well as multinodular hyperplasia $(87.5 \%, 14 / 16)$ specimens were EBNA2-positive, while the corresponding percentages for the adjacent normal tissues were $85.7 \%$ (12/14) and $93.8 \%(15 / 16)$, respectively. Sequence analysis confirmed the results. Interestingly, $L M P 1$ sequence frequency in nodular and adjacent normal thyroid tissue presented significant differ- 
ences compared to EBNA2 sequence frequency ( $\mathrm{P}=0.0015$ for nodular tissue, and $\mathrm{P}=0.0006$ for normal tissue). None of the samples contained the EBERI sequence (47).

In contrast to the studies mentioned above, negative results regarding the association between thyroid tumors and EBV have been reported (67-69). None of the 45 PTCs resected from patients in the southern part of Kyushu, Japan, and subjected to EBER1 ISH tested positive for EBV, despite the fact that a few infiltrating lymhoid cells detected in $1(2.2 \%)$ of the specimens, revealed EBER1 positivity (67). Additionaly, no significant positive signal for EBV was revealed by PCR, ISH for EBER, and IHC, performed in 12 cases (11 females/1 male) of oncocytic PTC with lymphoid stroma (Warthin-like tumor) (68). Positive PCR results of 1 sample, were not confirmed from repeat $\mathrm{PCR}$, or ISH, thus the sample was considered negative for EBV (68). Finally, all 16 benign thyroid tumor samples taken from 34 to 56 year-old female patients from Taiwan, used as controls in a study associating non-familial breast cancer with viral factors, scored negative for EBV using PCR and Southern hybridization (69).

The hypothesis of HSV1 and HSV2 association with thyroid tumors, was investigated analyzing the detection of viral DNA in both benign and malignant thyroid lesions, as well as the expression of nectin-1, a herpesvirus entry mediator, in thyroid tissues and cancer cell lines (29). Thyroid cancer cell susceptibility to HSV, and its associated molecular mechanisms, were explored in vitro (29). Thyroid samples from 109 patients (44 benign/65 malignant), including 43 PTCs, 16 FTCs, 6 anaplastic carcinomas, 30 follicular adenomas and 14 autoimmune thyroid disease specimens were examined (29). The presence of thyroid oncogene mutations was also sought in 73 thyroid tumors. Initialy, HSV DNA was amplified with PCR, followed by sequencing. Subsequent investigation included the detection of HSV proteins by immunohistochemical staining, and the detection of nectin-1 by in-cell western blot analysis. Collectively, HSV DNA was detected in 43/109 (39.4\%) of the samples. Considering benign lesions, HSV1 DNA was detected in 11/44 (25\%), while HSV2 DNA was detected less frequently (1/44-2\%). HSV DNA was much more frequently detected in malignant lesions $(31 / 65,47.7 \%$; $\mathrm{P}=0.0454)$, although HSV1 sequences had the same prevalence in benign and malignant lesions. PTCs and lymph node metastases harboured mostly HSV2 DNA, while HSV1 DNA was detected predominantly in FTCs. Oncogenic mutations were revealed in $70 \%(21 / 30)$ of the HSV-positive tumors, compared with $27.2 \%(12 / 44)$ of the HSV-negative tumors. Immunoreactivity was detected in 21/25 (84\%) of the HSV-positive samples investigated, restricted to epithelial cells, unlike stromal fibroblasts, endothelial cells and infiltrating lymphocytes, which were negative. As regards nectin-1, its expression has been shown to be increased in thyroid tumors, particularly papillary cancers compared to normal specimens, as well as in all thyroid cancer cell lines, and correlated with cancer cell susceptibility to HSV infection (29).

Unlike the results of the above-mentioned study, neither HSV1 nor HSV2 were detected in any of the 16 benign thyroid tumor samples, in the previously mentioned study reported by Tsai et al (69). The results were also negative for HHV8, while CMV was related to thyroid tumors $(\mathrm{P}<0.05)$, and its DNA was detected in $4 / 16(25 \%)$ of the specimens.

\section{Conclusions and future perspectives}

Data from the above-mentioned studies have revealed that thyroid cancer specimens harboured viral DNA and/or gene products, from both the polyoma (44-47) and herpes (47, 63-66) families. High sensitivity in DNA and RNA detection even in small biopsy samples has been achieved using PCR (40). Using a PCR-based assay to detect viral sequences though, needs extreme care, since the large number of PCR cycles to increase its sensitivity, renders it susceptible to false-positive results due to laboratory contamination (40). Moreover, a PCR-based assay, used to investigate the presence of a viral sequence in a tumor biopsy sample, may detect these sequences in normal cells contained in the sample, and thus makes the tumor appear positive for the investigated virus (40). ISH (63-66), IHC $(29,44,45,63,65,66)$ and Southern blotting $(44,69)$, used by studies reviewed in this review article, as well as in situ PCR, are less susceptible to contamination, while in situ methods discriminate between various cellular locations of the virus in tissue sections, avoiding false-positive results from adjacent infected normal cells (40). Furthermore, DNA sequencing analysis, performed by several studies that were included in this review $(29,44-47)$, confirmed the presence of the viral sequences detected, aiding in the accuracy of the individual studies.

PyVs in thyroid cancer. SV40 was detected in groups of specimens, including thyroid malignancies, PTC (44-46), medullary carcinoma (45), anaplastic carcinoma (45), benign thyroid lesions-Graves' disease (45), benign thyroid nodules (46), as well as normal thyroid tissues, adjacent to papillary and anaplastic cancers (45) and from patients with multinodular goiter (45). On the contrary, groups of specimens from both malignant thyroid disease, medullary cancer (44), benign lesions, toxic diffuse goiter, Hashimoto thyroiditis (44), as well as normal thyroid tissue, adjacent to PTCs $(44,46)$, adjacent to benign thyroid nodules (46), from the same studies, all scored negative for SV40.

A significant difference in the SV40 detection rate in similar histotype specimen groups was observed in 2 of the studies $(44,45)$, which was attributed to the higher sensitivity of PCR (45), compared to that of Southern blotting hybridization (44). As regards the study by Vivaldi et al (45), the SV40 detection rate in thyroid tumor samples was interestingly high, varying according to the degree of tumor differentiation, and this pattern also applied to the corresponding adjacent normal thyroid tissues, although there was statistical significance in the prevalence of SV40 Tag N-terminal coding sequences in each tumor specimen vs. the adjacent normal tissue. The detection rates were much lower for benign thyroid lesions and normal thyroid tissue adjacent to benign thyroid disease (45). The high prevalence of SV40 in both neoplastic and normal tissues, led the authors to hypothesize that viral infection potential spreads from neoplastic to adjacent normal tissue (45). The presence of SV40 in blood cells of healthy individuals, indicates that viral transfer to the thyroid gland may be thus achieved, and provides clues as to the long-term viral persistence in these cells in a latent state (45). Despite the use of the highly sensitive PCR, the prevalence of SV40 in PTC and benign nodules was relatively small in the third study (46). All normal thyroid 
tissues were negative, a feature that was attributed to the remote location of the specimens relative to the tumor (46), in contrast to the immediate proximity of the normal tissues relative to the tumor in the study by Vivaldi et al (45).

Although the presence of SV40 in tumor samples does not prove its causative role in the development of thyroid cancer, several findings indicate its potential role in thyroid carcinogenesis (44-46). Indeed, SV40 Tag has been shown to interfere with thyroid cell growth and differentiation, as evidenced by studies on transgenic mice, in which JC Tag under the transcriptional control of SV40 promoter, caused thyroid cell hyperplasia (70). Moreover, thyroid dedifferentiation and follicular cell proliferation, leading to the development of hyperplasia and adenocarcinomas, was achieved by genating transgenic mice that carried the SV40 Tag gene under the transcriptional control of the thyroid-specific thyroglobulin gene promoter (71). Furthermore, the insertion of the SV40 Tag gene in cultures of normal follicular cells, caused their escape from early mortality and loss of thyroid differentiated functions (72). Thyroid hormone receptor- $\alpha 1$ (TR $\alpha 1$ ), together with retinoid $\mathrm{X}$ receptor- $\alpha(\mathrm{RXR} \alpha)$ have been found to regulate the SV40 late promoter, and their regulators are hypothesized to block the transcription of the viral late genes until the onset of viral replication $(73,74)$. With a cell-specific mechanism, early genes, including Tag, are poorly transcribed in the presence of late gene overexpression, the amount of Tag molecules, viral DNA copies, and virions being thus reduced (75). TR $\alpha 1$ and $\mathrm{RXR} \alpha$ are active in follicular thyroid cells (76), while TR $\alpha 1$ is found inactive due to gene mutations in approximately $60 \%$ of PTCs (77). In such cases, low levels of SV40 DNA replication, Tag expression, as well as virion production in thyroid cancer cells may occur, as the viral late promoter is left uninhibited by inactive $\mathrm{TR} \alpha 1$ and $\operatorname{RXR} \alpha$ (45). All of the above data indicate that the transforming activity of SV40 may affect the thyroid gland during persistent infection.

The presence of BKV in thyroid cancer specimens, was investigated by only one study (47). Viral sequences were commonly found in both nodular, as well as in adjacent normal tissues. Thus, it was suggested that BKV 'infection' was a very early event, occurring apparently within normal tissue. No assumptions considering the oncogenic potential of the virus in thyroid cancer development were made, and a coexistence or 'endemicity' pattern, rather than a causal effect was instead suggested (47). Exposure of the thyroid gland to the virus, is probably achieved in the context of the viremic phase of infection by BKV (78).

Herpesviruses in thyroid cancer. The suggestion of the causal role of EBV in the development of B cell lymphoma, particularly in immunocompromized patients, formed the background of the 3 studies that investigated the role of the virus in thyroid lymphoma development in the Japanese $(63,64)$ and Hong Kong Chinese (65) population, respectively. Although it was concluded that EBV may play a role in a subset of thyroid lymphomas (65), and may specifically participate in the transformation of Hashimoto's thyroiditis, an entity known to play an important role in thyroid lymphoma development, to thyroid lymphoma (63), the activation of the virus in thyroid lymphoma is not common (64). The role of EBV in the development of thyroid lymphoma is further complicated by reports considering the incidence rates of EBV contact $(64,79)$. No obvious difference in the frequency of EBV exposure among individuals with thyroid lymphoma, Hashimoto's thyroiditis and simple goiter were found in Kuma Hospital (64), while higher EBV antibody titers were recorded in patients with thyroid lymphoma compared to those with Hashimoto's thyroiditis, whereas the latter frequently had higher titers compared to normal subjects (79). This finding, underscored the potential role of EBV in thyroid lymphoma development, either as a pathogenetic factor, or as a consequence of the disease (79). Investigation of proviral DNA in the nuclei of lymphoma cells, was suggested to prove the direct role of EBV infection in thyroid lymphoma development (79).

The presence of EBV in other histotypes of thyroid cancer remains controversial. Various studies have revealed either the absence of the virus from all cases of PTC (67), presence in the majority of samples of PTC (47), and presence in $100 \%$ of both papillary and undifferentiated carcinomas, although EBV mRNA and protein expression was much more prominent in undifferentiated carcinoma samples, a fact that led to the assumption of EBV contribution to tumor dedifferentiation (66). Inconsistency between PCR and the less sensitive ISH method characterized the results of the investigation of Warthin-like tumor for EBV (68). These were attributed to a potential falsely positive PCR, resulting from the presence of circulating lymphocytes that carried EBV in the tumor sample, since one in $10^{6}$ circulating B lymphocytes may carry EBV (68). The difference between the strongly positive results of Shimakage et al (66), and the negative results of Kijima et al (67) and Ludvíková et al (68), both of whom performed ISH using the EBER1 probe, was attributed to the different sensitivity of the probes used (66). In accordance to the results of Shimakage et al (66), considering the presence of EBV in PTC samples, Stamatiou et al (47) also tested adjacent normal thyroid samples using PCR, which showed comparatively high positivity for EBV. Interestingly, the detection rate of the 3 EBV genes investigated, differed significantly (47). The results were verified with DNA sequencing and a potential viral spread from normal to cancerous tissue was supposed (47).

Tumor B cell inflamatory infiltrates may play the role of viral reservoirs, as regards the infection of thyroid epithelial cells by EBV, a lymphotropic virus (68). The proven fusion of infected B cells with epithelial cells (80), as well as the observation that the in vitro infection of epithelial cell lines by EBV was only possible during coculture with EBV-infected B cells, but impossible using cell-free EBV (81), offer a potential mechanism of viral transfer between cell types.

Furthermore, it should be noted that despite the fact that studies investigating the presence of EBNA in oropharyngeal epithelial cells obtained from throat washings of healthy adult volunteers, recorded no differences regarding EBV positivity between Far Eastern populations, Osaka, Japan (27\%), and Caucasians, United States (22\%) (82), reports associating EBV with tumors, depend on regional and/or ethnic background, occuring in Asians but not in Caucasians (83). Since the majority of the above-mentioned studies refer to Far Eastern populations (63-67), locality features should be considered and larger studies with random patient selection performed. 
HSV DNA and protein was detected in thyroid cancer specimens with high frequency, and their presence related to the activation of virus-inducible signaling in thyroid cells (29). Furthermore, thyroid cancer cell lines were permissive to HSV infection, while inhibition of mitogenic signaling, decreased their susceptibility to the virus (29). Activation of nuclear factor (NF)-kB and p-AKT signaling, usually associated with oncogene mutations, was shown to occur due to HSV infection, while the activation of the RAS/MEK/MAPK pathway, which characterizes thyroid cancer, contributes to active HSV replication in thyroid cancer cells (29). Increased nectin-1 expression in thyroid tumors relative to normal thyroid tissue, its expression being further increased during tumor progression, may provide an explanation for the increased frequency of HSV in thyroid tumors, its level correlating with HSV infection susceptibility in cancer cell lines (29). The specific propensity of FTCs and PTCs toward HSV1 and HSV2, respectively, analogous to the specific tropism of different neurons in the same ganglion to HSV1 and HSV2, may underlie the tumor-specific patterns of signal transduction (84).

Considering the presence of CMV in benign thyroid tumors, further studies need to be performed, to clarify its association with human thyroid tissues.

On the whole, after reviewing the studies investigating the presence of both viral DNA and gene products of the herpes and polyomaviridae families in thyroid cancer tissues, there seems to be an association of both virus families with thyroid malignancies. Caution should be undertaken when interpreting these virological data and in conclusion making. Since members of both viral families are ubiquitous among humans, the role of these viruses as causal factors in thyroid tumorigenesis cannot be proven by the mere detection of viral sequences in tumor samples, as the viruses may be attracted to the tumor by the specific milieu of growth factors and cytokines of the tumor environment. This also applies to the presence of specific virus-directed antibodies, that may just represent the trace of passage of a virus and its clearance from the body. On the other hand, the absence of viral markers is not sufficient to exclude the viral contribution in cancer development, since the triggering infection may have taken place many years previously, and viral interaction with the specific genetic background of the host may have quiescently played a role in host disease development. As regards their potential contribution to the thyroid oncogenic process, other genetic and environmental co-factors should definitely play a role in disease development, apart from the life-term persistent infection associated with members of both viral families reviewed herein, since the incidence of the neoplastic disorders is far smaller than the prevalence of the investigated viruses in the population, as is the case with EBV infection (85).

Since the fulfillment of Koch's four postulates, the formal criteria for microbial disease causation, has proven difficult for any of the oncogenic viruses discovered to date (56), the oncogenicity of herpes and PyVs in thyroid cancer remains controversial. Genomic integration, instead of mere detection of viral genomic sequences or proteins, proposed as a means of clarification of the association between viruses and cancer (56), may potentially provide an answer. Definitely, more convincing evidence is required, and the present review is just the beginning of a long research journey in the clarification of whether these viruses are responsible for thyroid cancer development, or just represent innocent bystanders.

\section{References}

1. Bhaijee F and Nikiforov YE: Molecular analysis of thyroid tumors. Endocr Pathol 22: 126-133, 2011.

2. Nikiforov YE: Molecular analysis of thyroid tumors. Mod Pathol 24 (Suppl 2): S34-S43, 2011.

3. World Health Organization Classification of Tumors: Pathology and Genetics of Tumours of Endocrine Organs. DeLellis RA, Lloyd RV, Heitz PU and Eng C (eds). IARC Press, Lyon, 2004.

4. Nikiforov YE: Thyroid tumors: classification and general considerations. In: Diagnostic pathology and molecular genetics of the thyroid. Nikiforov YE, Biddinger PW and Thompson LDR (eds). Lippincott Williams \& Wilkins, Baltimore, MD, pp94-102, 2009.

5. Gulcelik MA, Gulcelik NE, Kuru B, Camlibel M and Alagol H: Prognostic factors determining survival in differentiated thyroid cancer. J Surg Oncol 96: 598-604, 2007.

6. Roy M, Chen H and Sippel RS: Current understanding and management of medullary thyroid cancer. Oncologist 18: 1093-1100, 2013.

7. Hundahl SA, Cady B, Cunningham MP, Mazzaferri E, McKee RF, Rosai J, Shah JP, Fremgen AM, Stewart AK and Hölzer S: Initial results from a prospective cohort study of 5583 cases of thyroid carcinoma treated in the united states during 1996. U.S. and German Thyroid Cancer Study Group. An American College of Surgeons Commission on Cancer Patient Care Evaluation study. Cancer 89: 202-217, 2000.

8. Thyroid Cancer Treatment (PDQR): Medullary Thyroid Cancer. http://www.cancer.gov./cancertopics/pdq/treatment/ thyroid/HealthProfessional/page7. Accessed July 29, 2013.

9. Desailloud R and Hober D: Viruses and thyroiditis: an update. Virol J 6: 5, 2009.

10. Aozasa K, Ueda T, Katagiri S, Matsuzuka F, Kuma K and Yonezawa T: Immunologic and immunohistologic analysis of 27 cases with thyroid lymphomas. Cancer 60: 969-973, 1987.

11. Ron E and Schneider A: Thyroid cancer. In: Cancer Epidemiology and Prevention. Schottenfeld D and Fraumeni J (eds). Oxford University Press, New York, NY, pp975-994, 2006.

12. Simard EP, Ward EM, Siegel R and Jemal A: Cancers with increasing incidence trends in the United States: 1999 through 2008. CA Cancer J Clin 62: 118-128, 2012.

13. Davies L and Welch HG: Increasing incidence of thyroid cancer in the United States, 1973-2002. JAMA 295: 2164-2167, 2006.

14. Davies L, Ouellette M, Hunter M and Welch HG: The increasing incidence of small thyroid cancers: where are the cases coming from? Laryngoscope 120: 2446-2451, 2010.

15. Aschebrook-Kilfoy B, Ward MH, Sabra MM and Devesa SS: Thyroid cancer incidence patterns in the United States by histologic type, 1992-2006. Thyroid 21: 125-134, 2011.

16. Chen AY, Jemal A and Ward EM: Increasing incidence of differentiated thyroid cancer in the United States, 1988-2005. Cancer 115: 3801-3807, 2009.

17. Enewold L, Zhu K, Ron E, Marrogi AJ, Stojadinovic A, Peoples GE and Devesa SS: Rising thyroid cancer incidence in the United States by demographic and tumor characteristics, 1980-2005. Cancer Epidemiol Biomarkers Prev 18: 784-791, 2009.

18. Ahmed R, Al-Shaikh S and Akhtar M: Hashimoto thyroiditis: a century later. Adv Anat Pathol 19: 181-186, 2012.

19. Holm LE, Blomgren $H$ and Löwhagen T: Cancer risks in patients with chronic lymphocytic thyroiditis. N Engl J Med 312: 601-604, 1985.

20. Kimura ET, Nikiforova MN, Zhu Z, Knauf JA, Nikiforov YE and Fagin JA: High prevalence of BRAF mutations in thyroid cancer: Genetic evidence for constitutive activation of the RET/PTC-RAS-BRAF signaling pathway in papillary thyroid carcinoma. Cancer Res 63: 1454-1457, 2003.

21. Soares P, Trovisco V, Rocha AS, Lima J, Castro P, Preto A Máximo V, Botelho T, Seruca R and Sobrinho-Simões M: BRAF mutations and RET/PTC rearrangements are alternative events in the etiopathogenesis of PTC. Oncogene 22: 4578-4580, 2003.

22. Frattini M, Ferrario C, Bressan P, Balestra D, De Cecco L, Mondellini P, Bongarzone I, Collini P, Gariboldi M, Pilotti S, et al: Alternative mutations of BRAF, RET and NTRK1 are associated with similar but distinct gene expression patterns in papillary thyroid cancer. Oncogene 23: 7436-7440, 2004. 
23. Adeniran AJ, Zhu Z, Gandhi M, Steward DL, Fidler JP, Giordano TJ, Biddinger PW and Nikiforov YE: Correlation between genetic alterations and microscopic features, clinical manifestations, and prognostic characteristics of thyroid papillary carcinomas. Am J Surg Pathol 30: 216-222, 2006

24. Kroll TG, Sarraf P, Pecciarini L, Chen CJ, Mueller E, Spiegelman BM and Fletcher JA: PAX8-PPARgammal fusion oncogene in human thyroid carcinoma (corrected). Science 289 : 1357-1360, 2000.

25. Nikiforova MN, Lynch RA, Biddinger PW, Alexander EK, Dorn GW II, Tallini G, Kroll TG and Nikiforov YE: RAS point mutations and PAX8-PPAR gamma rearrangement in thyroid tumors: evidence for distinct molecular pathways in thyroid follicular carcinoma. J Clin Endocrinol Metab 88: 2318-2326, 2003.

26. Fagin JA and Mitsiades N: Molecular pathology of thyroid cancer: Diagnostic and clinical implications. Best Pract Res Clin Endocrinol Metab 22: 955-969, 2008.

27. Ricarte-Filho JC, Ryder M, Chitale DA, Rivera M, Heguy A, Ladanyi M, Janakiraman M, Solit D, Knauf JA, Tuttle RM, et al: Mutational profile of advanced primary and metastatic radioactive iodine-refractory thyroid cancers reveals distinct pathogenetic roles for BRAF, PIK3CA, and AKT1. Cancer Res 69: 4885-4893, 2009.

28. Vasko V, Espinosa AV, Scouten W, He H, Auer H, Liyanarachchi S Larin A, Savchenko V, Francis GL, de la Chapelle A, et al: Gene expression and functional evidence of epithelial-to-mesenchymal transition in papillary thyroid carcinoma invasion. Proc Nat Acad Sci USA 104: 2803-2808, 2007

29. Jensen K, Patel A, Larin A, Hoperia V, Saji M, Bauer A, Yim K, Hemming V and Vasko V: Human herpes simplex viruses in benign and malignant thyroid tumours. J Pathol 221: 193-200, 2010.

30. Kaul R, Murakami M, Lan K, Choudhuri T and Robertson ES: EBNA3C can modulate the activities of the transcription factor Necdin in association with metastasis suppressor protein Nm23-H1. J Virol 83: 4871-4883, 2009.

31. Horikawa T, Yang J, Kondo S, Yoshizaki T, Joab I, Furukawa M and Pagano JS: Twist and epithelial-mesenchymal transition are induced by the EBV oncoprotein latent membrane protein 1 and are associated with metastatic nasopharyngeal carcinoma. Cancer Res 67: 1970-1978, 2007.

32. Tsuji A, Wakisaka N, Kondo S, Murono S, Furukawa M and Yoshizaki T: Induction of receptor for advanced glycation end products by EBV latent membrane protein 1 and its correlation with angiogenesis and cervical lymph node metastasis in nasopharyngeal carcinoma. Clin Cancer Res 14: 5368-5375, 2008.

33. Strauss R, Sova P, Liu Y, Li ZY, Tuve S, Pritchard D Brinkkoetter P, Möller T, Wildner O, Pesonen S, et al: Epithelial phenotype confers resistance of ovarian cancer cells to oncolytic adenoviruses. Cancer Res 69: 5115-5125, 2009.

34. Zhang X, Zhang Z, Zheng B, He Z, Winberg G and Ernberg I: An update on viral association of human cancers. Arch Virol 158 $1433-1443,2013$

35. McLaughlin-Drubin ME and Munger K: Viruses associated with human cancer. Biochim Biophys Acta 1782: 127-150, 2008

36. Feng H, Shuda M, Chang Y and Moore PS: Clonal integration of a polyomavirus in human Merkel cell carcinoma. Science 319 : 1096-1100, 2008.

37. Dalianis T and Hirsch HH: Human polyomaviruses in disease and cancer. Virology 437: 63-72, 2013.

38. Gjoerup $\mathrm{O}$ and Chang Y: Update on human polyomaviruses and cancer. Adv Cancer Res 106: 1-51, 2010

39. Cheng J, DeCaprio JA, Fluck MM and Schaffhausen BS: Cellular transformation by Simian Virus 40 and Murine Polyoma Virus T antigens. Semin Cancer Biol 19: 218-228, 2009.

40. Abend JR, Jiang M and Imperiale MJ: BK virus and human cancer: innocent until proven guilty. Semin Cancer Biol 19: 252-260, 2009.

41. Imperiale MJ: The human polyomaviruses, BKV and JCV: Molecular pathogenesis of acute disease and potential role in cancer. Virology 267: 1-7, 2000.

42. Imperiale MJ: Oncogenic transformation by the human polyomaviruses. Oncogene 20: 7917-7923, 2001.

43. Tognon M, Corallini A, Martini F, Negrini $M$ and Barbanti-Brodano G: Oncogenic transformation by BK virus and association with human tumors. Oncogene 22: 5192-5200, 2003.

44. Pacini F, Vivaldi A, Santoro M, Fedele M, Fusco A, Romei C, Basolo F and Pinchera A: Simian virus 40-like DNA sequences in human papillary thyroid carcinomas. Oncogene 16: 665-669, 1998 .
45. Vivaldi A, Pacini F, Martini F, Iaccheri L, Pezzetti F, Elisei R, Pinchera A, Faviana P, Basolo F and Tognon M: Simian virus 40-like sequences from early and late regions in human thyroid tumors of different histotypes. J Clin Endocrinol Metab 88: 892-899, 2003

46. Ozdarendeli A, Camci C, Aygen E, Kirkil C, Toroman ZA, Dogru O and Doymaz MZ: SV40 in human thyroid nodules. J Clin Virol 30: 337-340, 2004

47. Stamatiou D, Derdas SP, Symvoulakis EK, Sakorafas GH,Zoras O and Spandidos DA: Investigation of BK virus, Epstein-Barr virus and human papillomavirus sequences in postoperative thyroid gland specimens. Int J Biol Markers 30: e104-e110, 2015.

48. Sweet BH and Hilleman MR: The vacuolating virus, S.V. 40 Proc Soc Exp Biol Med 105: 420-427, 1960.

49. Grinde B: Herpesviruses: latency and reactivation - viral strategies and host response. J Oral Microbiol 5: 2013.

50. Dreyfus DH: Herpesviruses and the microbiome. J Allergy Clin Immunol 132: 1278-1286, 2013.

51. Farooq AV and Shukla D: Herpes simplex epithelial and stromal keratitis: An epidemiologic update. Surv Ophthalmol 57: 448-462, 2012

52. Rozenberg F, Deback C and Agut H: Herpes simplex encephalitis: From virus to therapy. Infect Disord Drug Targets 11: 235-250, 2011.

53. Zerr DM, Meier AS, Selke SS, et al: A population-based study of primary human herpesvirus 6 infection. N Engl J Med 352: 768-776, 2005 .

54. Townsend CL, Forsgren M, Ahlfors K, Ivarsson SA, Tookey PA and Peckham CS: Long-term outcomes of congenital cytomegalovirus infection in Sweden and the United Kingdom. Clin Infect Dis 56: 1232-1239, 2013.

55. Boshoff $\mathrm{C}$ and Weiss R: AIDS-related malignancies. Nat Rev Cancer 2: 373-382, 2002.

56. Sarid R and Gao SJ: Viruses and human cancer: From detection to causality. Cancer Lett 305: 218-227, 2011

57. Kutok JL and Wang F: Spectrum of Epstein-Barr virus-associated diseases. Annu Rev Pathol 1: 375-404, 2006.

58. Deshmane SL and Fraser NW: During latency, herpes simplex virus type 1 DNA is associated with nucleosomes in a chromatin structure. J Virol 63: 943-947, 1989.

59. Wilson AC and Mohr I: A cultured affair: HSV latency and reactivation in neurons. Trends Microbiol 20: 604-611, 2012.

60. Given D, Yee D, Griem K and Kieff E: DNA of Epstein-Barr virus. V. Direct repeats of the ends of Epstein-Barr virus DNA. J Virol 30: 852-862, 1979.

61. Cai X, Schafer A, Lu S, Biello JP, Desrosiers RC, Edwards R, Raab-Traub N and Cullen BR: Epstein-Barr virus microRNAs are evolutionarily conserved and differentially expressed. PLoS Pathog 2: e23. Epub 2006.

62. Fuentes-González AM, Contreras-Paredes A, Manzo-Merino J and Lizano M: The modulation of apoptosis by oncogenic viruses. Virol J 10: 182, 2013.

63. Takahashi K, Kashima K, Daa T, Yokoyama S, Nakayama I and Noguchi S: Contribution of Epstein-Barr virus to development of malignant lymphoma of the thyroid. Pathol Int 45: 366-374, 1995.

64. Tomita Y, Ohsawa M, Kanno H, Matsuzuka F, Kuma K and Aozasa K: Sporadic activation of Epstein-Barr virus in thyroid lymphoma. Leuk Lymphoma 19: 129-134, 1995.

65. Lam KY, Lo CY, Kwong DL, Lee J and Srivastava G: Malignant lymphoma of the thyroid. A 30-year clinicopathologic experience and an evaluation of the presence of Epstein-Barr virus. Am J Clin Pathol 112: 263-270, 1999.

66. Shimakage M, Kawahara K, Sasagawa T, Inoue H, Yutsudo M, Yoshida A and Yanoma S: Expression of Epstein-Barr virus in thyroid carcinoma correlates with tumor progression. Hum Pathol 34: 1170-1177, 2003.

67. Kijima Y, Hokita S, Takao S, Baba M, Natsugoe S, Yoshinaka H, Aridome K, Otsuji T, Itoh T, Tokunaga M, et al: Epstein-Barr virus involvement is mainly restricted to lymphoepithelial type of gastric carcinoma among various epithelial neoplasms. J Med Virol 64: 513-518, 2001.

68. Ludvíková M, Ryska A, Korabecná M, Rydlová M and Michal M: Oncocytic papillary carcinoma with lymphoid stroma (Warthin-like tumour) of the thyroid: a distinct entity with favourable prognosis. Histopathology 39: 17-24, 2001.

69. Tsai JH, Tsai CH, Cheng MH, Lin SJ, Xu FL and Yang CC: Association of viral factors with non-familial breast cancer in Taiwan by comparison with non-cancerous, fibroadenoma, and thyroid tumor tissues. J Med Virol 75: 276-281, 2005. 
70. Feigenbaum L, Hinrichs SH and Jay G: JC virus and simian virus 40 enhancers and transforming proteins: Role in determining tissue specificity and pathogenicity in transgenic mice. $\mathrm{J}$ Virol 66: 1176-1182, 1992.

71. Ledent C, Dumont J, Vassart G and Parmentier M: Thyroid adenocarcinomas secondary to tissue-specific expression of simian virus-40 large T-antigen in transgenic mice. Endocrinology 129: 1391-1401, 1991.

72. Bond JA, Oddweig Ness G, Rowson J, Ivan M, White D and Wynford-Thomas D: Spontaneous de-differentiation correlates with extended lifespan in transformed thyroid epithelial cells: An epigenetic mechanism of tumour progression? Int J Cancer 67: 563-572, 1996

73. Zuo F and Mertz JE: Simian virus 40 late gene expression is regulated by members of the steroid/thyroid hormone receptor superfamily. Proc Natl Acad Sci USA 92: 8586-8590, 1995.

74. Zuo F, Kraus RJ, Gulick T, Moore DD and Mertz JE: Direct modulation of simian virus 40 late gene expression by thyroid hormone and its receptor. J Virol 71: 427-436, 1997.

75. Farrell ML and Mertz JE: Cell type-specific replication of simian virus 40 conferred by hormone response elements in the late promoter. J Virol 76: 6762-6770, 2002.

76. schmutzler C, Brtko J, Winzer R, Jakobs TC, Meissner-Weigl J, Simon D, Goretzki PE and Köhrle J: Functional retinoid and thyroid hormone receptors in human thyroid-carcinoma cell lines and tissues. Int J Cancer 76: 368-376, 1998.

77. Puzianowska-Kuznicka M, Krystyniak A, Madej A, Cheng SY and Nauman J: Functionally impaired TR mutants are present in thyroid papillary cancer. J Clin Endocrinol Metab 87: 1120-1128, 2002 .
78. Hirsch HH and Steiger J: Polyomavirus BK. Lancet Infect Dis 3: 611-623, 2003.

79. Matsubayashi S, Tamai H, Morita T, Mori K, Katsuki T, Matsuzuka F, Kuma K and Nagataki S: Malignant lymphoma of the thyroid and Epstein-Barr virus. Endocrinol Jpn 36: 343-348, 1989.

80. Bayliss GJ and Wolf H: An Epstein - Barr virus early protein induces cell fusion. Proc Natl Acad Sci USA 78: 7162-7165, 1981.

81. Yoshiyama H, Imai S, Shimizu N and Takada K: Epstein-Barr virus infection of human gastric carcinoma cells: implication of the existence of a new virus receptor different from CD21. J Virol 71: 5688-5691, 1997.

82. Sixbey JW, Shirley P, Chesney PJ, Buntin DM and Resnick L: Detection of a second widespread strain of Epstein-Barr virus. Lancet 2: 761-765, 1989.

83. Chan JK, Hui PK, Yip TT, Tsang WY, Law CK, Poon YF and Ma VW: Detection of Epstein-Barr virus only in lymphoepithelial carcinomas among primary carcinomas of the lung. Histopathology 26: 576-578, 1995.

84. Margolis TP, Imai Y, Yang L, Vallas V and Krause PR: Herpes simplex virus type 2 (HSV-2) establishes latent infection in a different population of ganglionic neurons than HSV-1: role of latency-associated transcripts. J Virol 81: 1872-1878, 2007.

85. Shah KM and Young LS: Epstein-Barr virus and carcinogenesis: Beyond Burkitt's lymphoma. Clin Microbiol Infect 15: 982-988, 2009. 